ESAIM: PROCEEDINGS, December 2012, Vol. 38, p. 78-98

F. Coquel, M. Gutnic, P. Helluy, F. Lagoutière, C. Rohde, N. Seguin, Editors

\title{
SEDIMENT TRANSPORT MODELLING : RELAXATION SCHEMES FOR SAINT-VENANT - EXNER AND THREE LAYER MODELS
}

\author{
Emmanuel Audusse ${ }^{1}$, Christophe Berthon ${ }^{2}$, Christophe Chalons ${ }^{3}$, Olivier \\ Delestre $^{4}$, Nicole Goutal ${ }^{5}$, Magali Jodeau ${ }^{6}$, Jacques Sainte-Marie ${ }^{7}$, Jan \\ GiESSELMANN $^{8}$ AND GEORGES SADAKA ${ }^{9}$
}

\begin{abstract}
In this note we are interested in the modelling of sediment transport phenomena. We mostly focus on bedload transport and we do not consider suspension sediment processes. We first propose a numerical scheme for the classical Saint-Venant - Exner model. It is based on a relaxation approach for the whole system and it works with all sediment flux function. The stability of the scheme is investigated and some numerical tests are proposed. We exhibit that this coupled approach is more stable than the splitting approach that is mostly used in industrial softwares. Then we derive an original three layers model in order to overcome the difficulties that are encountered when using the classical Exner approach and we present a related relaxation model.
\end{abstract}

\section{Sediment transport modelling : A major issue}

In rivers, mean sediment discharge may represent several hundred cubic meters of gravels or silt per year. Therefore, the sediments must be taken into account in order to predict the river bed evolutions. For hydroelectricity managers sediment transport modelling is a major issue. Dams stop water and consequently impact sediment transport, as well as sediment deposition may disturb water intakes or bottom gate opening, for example see Fig. 1. Besides, in some rivers like the Loire river, water intakes of nuclear power station may be protected against the deposition of sand. In order to understand sediment transport dynamics and to suggest managing solutions, EDF has been working on sediment transport modelling tools for bedload and suspension sediment transport. This work focuses on the modelling of bedload transport which refers to gravel transport and pushes aside the transport of fine sediments by suspension.

Up to now, one very classical approach is to approximate the solid phase equation by a simplified one : the well-known Exner equation [10]. The Exner equation is obtained by writing a mass conservation on the solid

${ }^{1}$ BANG Project, INRIA-Paris-Rocquencourt \& LAGA, University Paris 13 Nord, France, e-mail : audusse@math.univ-paris13.fr

2 Lab. J. Leray, University of Nantes, France, e-mail : christophe.berthon@math.univ-nantes.fr

${ }^{3}$ LJLL, University Paris Diderot, France, e-mail : chalons@math.jussieu.fr

${ }^{4}$ Lab. J.A. Dieudonné \& EPU Sofia Nice, University of Nice, France, e-mail : delestre@polytech.unice.fr

${ }^{5}$ EDF R\&D \& Saint-Venant Lab., France, e-mail : nicole.goutal@edf.fr

${ }^{6}$ EDF R\&D, France, e-mail : magali.jodeau@edf.fr

7 BANG Project, INRIA-Paris-Rocquencourt \& CETMEF, France, e-mail : jacques.sainte-marie@inria.fr

8 IAANS, University of Stuttgart, Germany, e-mail : jan.giesselmann@mathematik.uni-stuttgart.de

${ }^{9}$ LAMFA, University of Picardie, France, e-mail : georges.sadaka@u-picardie.fr

(C) EDP Sciences, SMAI 2012 


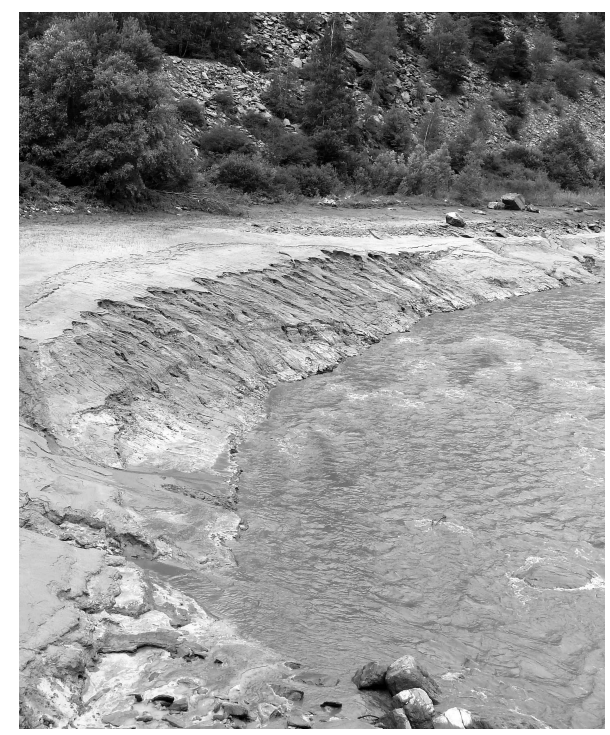

Figure 1. Example of silt deposition pattern in reservoirs (water flows from the back to the front).

phase in interaction with the fluid. There is no dynamic effect in the solid phase

$$
\rho_{s}(1-p) \frac{\partial Z}{\partial t}+\frac{\partial Q_{s}}{\partial x}=0
$$

where $Z$ is the bed elevation, $Q_{s}$ the bed load, $p$ the porosity of the gravel bed. The bed load may be expressed by empirical formulae of the form

$$
Q_{s}=A_{g}(u)|u|^{m-1} u
$$

where $u$ is the velocity in the fluid (Grass [13] formula) or $Q_{s}=f\left(\tau_{b}\right)$ where $\tau_{b}$ is the boundary shear stress (see for example Meyer-Peter and Muller [18], Einstein [8] or Engelund and Fredsoe [9] formulas).

The classical fluid model is the shallow water equations. It is coupled with the solid phase by the bottom level evolution. The coupled model stands ${ }^{1}$ :

$$
\begin{aligned}
\frac{\partial H}{\partial t}+\frac{\partial Q}{\partial x} & =0 \\
\frac{\partial Q}{\partial t}+\frac{\partial}{\partial x}\left(\frac{Q^{2}}{H}+\frac{g}{2} H^{2}\right) & =-g H \frac{\partial Z}{\partial x}, \\
\rho_{s}(1-p) \frac{\partial Z}{\partial t}+\frac{\partial Q_{s}}{\partial x} & =0,
\end{aligned}
$$

where $Q=H u$ is the water discharge and $H$ the water height.

There are two major ways to solve this three-equation system that we refer in the following as the external and the internal coupling methods. The strategy developed at EDF-RD is based on an external coupling method. Firstly, the hydraulic part of the system is solved by using the software MASCARET [12] and then the computed fluid quantities are sent to the software COURLIS [4] that solves the Exner equation. The fluid part and the solid one are coupled through the time evolution of the bottom level. In the best way, the shallow-water

\footnotetext{
${ }^{1}$ For simplicity's sake, equations are written for the one dimension problem in rectangular channels
} 
equations and the Exner equation are coupled at each time step without local iterations. EDF actual modelling tools are used in complex cases, for example the draw-down of a reservoir with a steep slope and deep zones. Engineers are confronted with the limitation of the codes. Fig 2 is an example of instabilities observed in the case of a dam break calculation using an external coupling strategy (0.3s after dam break). This result is in agreement with [7] where the authors show that, with the external coupling method, the instabilities cannot be always avoided in supercritical regions. Thus alternative approaches must be investigated.
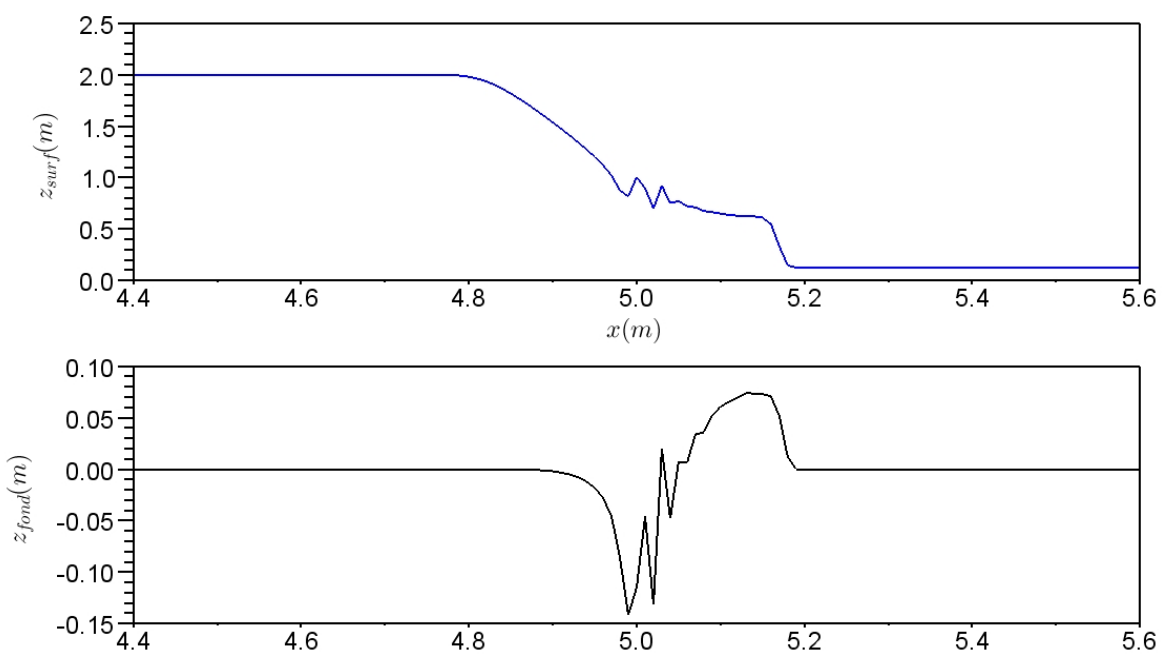

FiguRE 2. Dam break over a movable bottom with a splitting approach - Free surface (top) and Bottom topography (bottom)

In this note, we investigate two different approaches. First we consider the internal coupling approach that consists in solving the system (2)-(4) at once with three unknowns $H, Q$ and $Z$. We then have a hyperbolic system to solve and some specific finite volume schemes have been recently developed, see $[2,6]$. For stiff cases this approach seems to be more robust than the uncoupled one [7]. Here we propose a relaxation approach of the model that allows us to deal with a large class of sediment fluxes and we construct the related relaxation solver. For an introduction to the relaxation framework, we refer the reader to $[5,15]$. Second we go one step further and we derive a new three layers model that is able to propose a more accurate modelling of the phenomenon than the classical Exner approach. This new model is similar to some models introduced in [20]. We also propose a relaxation approach for this new model.

The outline of the paper is the following. In Section 2, we introduce a relaxation approach to derive a stable numerical solver for the classical SW-Exner model (2)-(4). Then we propose some numerical test cases in Section 3. In Sections 4 and 5 we derive the new three-layer model and we propose a related relaxation model in Section 6 .

\section{A Relaxation solver for the Saint-Venant - Exner model}

In this section, we consider the classical Saint-Venant - Exner model (2)-(4). Considering the internal coupling framework, we first propose a relaxation approach for this model and then we construct the related numerical solver. A paragraph is devoted to the stability of the relaxation model and to the well-posedness of the relaxation solver. This work has two main motivations : first, to construct a stable solver for the numerical simulation of model (2)-(4) ; second, to introduce the main ideas that will be used to derive the relaxation model associated to the three layers model that is presented in the second part of the paper. The relaxation 
framework that we consider in this Section was first introduced in [17].

The hyperbolic nature of the SW-Exner model (2)-(4) strongly depends on the formula that is chosen for the sediment flux $Q_{s}[7]$. Moreover the computation of the eigenvalues (that are needed if we want to compute an approximate Riemann solver) of the system needs for iterative strategies except for very particular choices of $Q_{s}$. Here we overcome this difficulty by considering a relaxation model where we relax the sediment flux. We also relax the fluid pressure as it is usually done for classical shallow water or Euler systems. It leads to the introduction of two new auxiliary variables denoted $\Pi$ for the pressure and $\Omega$ for the sediment flux. The relaxation system finally stands as a larger but more suitable hyperbolic system since the auxiliary equations are chosen such that all the fields are linearly degenerate. The method is well-described in [5] where in addition a large number of classical hyperbolic solver are interpreted as relaxation schemes. In our case we finally obtain the following model, see also [17]

$$
\begin{aligned}
\frac{\partial H}{\partial t}+\frac{\partial H u}{\partial x} & =0 \\
\frac{\partial H u}{\partial t}+\frac{\partial}{\partial x}\left(H u^{2}+\Pi\right)+g H \frac{\partial Z}{\partial x} & =0 \\
\frac{\partial \Pi}{\partial t}+u \frac{\partial \Pi}{\partial x}+\frac{a^{2}}{H} \frac{\partial u}{\partial x} & =\frac{1}{\lambda}\left(\frac{g H^{2}}{2}-\Pi\right) \\
\frac{\partial Z}{\partial t}+\frac{\partial \Omega}{\partial x} & =0 \\
\frac{\partial \Omega}{\partial t}+\left(\frac{b^{2}}{H^{2}}-u^{2}\right) \frac{\partial Z}{\partial x}+2 u \frac{\partial \Omega}{\partial x} & =\frac{1}{\lambda}\left(Q_{s}-\Omega\right)
\end{aligned}
$$

where $\lambda$ is a small positive parameter. The parameters $a$ and $b$ will be chosen later to ensure the stability of the model. The main properties of system (5)-(9) are the following

- It obviously tends (at least formally) to the original SW-Exner model (2)-(4) when $\lambda$ tends to zero.

- It is always hyperbolic and the eigenvalues are are ordered and does not depend on the special choice of the sediment flux $Q_{s}$.

- All the fields are linearly degenerate and the Riemann problem is easy to solve.

\subsection{Construction of the relaxation solver}

We can write system (5)-(9) on the quasi-linear form

$$
\partial_{t} V+A(V) \partial_{x} V=S_{\lambda}(V)
$$

with

$$
V=\left(\begin{array}{c}
H \\
u \\
\Pi \\
Z \\
\Omega
\end{array}\right), \quad A(V)=\left(\begin{array}{ccccc}
u & H & 0 & 0 & 0 \\
0 & u & 1 / H & g & 0 \\
0 & a^{2} / H & u & 0 & 0 \\
0 & 0 & 0 & 0 & 1 \\
0 & 0 & 0 & \frac{b^{2}}{H^{2}}-u^{2} & 2 u
\end{array}\right), \quad S_{\lambda}(V)=\left(\begin{array}{c}
0 \\
0 \\
\frac{1}{\lambda}\left(\frac{g H^{2}}{2}-\Pi\right) \\
0 \\
\frac{1}{\lambda}\left(Q_{s}-\Omega\right)
\end{array}\right)
$$

Our relaxation solver is based on a time splitting strategy that can be written in two steps. First, starting from the physical quantities $\left(H^{n}, u^{n}, Z^{n}\right)$, we compute the auxiliary quantities $\left(\Pi^{n}, \Omega^{n}\right)$ by

$$
\Pi^{n}=\frac{g\left(H^{n}\right)^{2}}{2}, \quad \Omega^{n}=Q_{s}\left(H^{n}, u^{n}\right)
$$


It can be interpreted as the solution of the following system of ODEs

$$
\partial_{t} V=S_{\lambda}(V)
$$

where we formally consider that $\lambda=0$. Second we consider the homogeneous hyperbolic system

$$
\partial_{t} V+A(V) \partial_{x} V=0
$$

and we compute the numerical solution at the next time step by using a Godunov scheme. To do this, we have to compute the solution $V_{i+1 / 2}^{n}$ of the associated Riemann problem at each interface

$$
V_{i+1 / 2}^{n}(0, x)=\left\{\begin{array}{lll}
V_{i} & \text { if } & x<x_{i+1 / 2} \\
V_{i+1} & \text { if } \quad x \geq x_{i+1 / 2}
\end{array}\right.
$$

The CFL condition is fixed to ensure that these interface Riemann problems do not interact

$$
\Delta t^{n} \leq \frac{\Delta x}{\max _{i} \rho\left(A_{i}^{n}\right)}
$$

where $\rho(A)$ denotes the largest absolute value of the eigenvalues of matrix $A$. Then we can define a global solution by

$$
\forall(t, x) \in\left[0, \Delta t^{n}\right] \times \mathbb{R} \quad V_{R}^{n}(t, x)=V_{i+1 / 2}^{n}(t, x) \chi_{\left[x_{i}, x_{i+1}\right]}(x)
$$

where $\chi_{I}$ denotes the characteristic function of the interval $I$. Finally the numerical solution $V_{i}^{n+1}$ at the next time step is computed by considering the mean value of this exact solution on the cell $C_{i}$ at time $\Delta t^{n}$, i.e.

$$
V_{i}^{n+1}=\frac{1}{x_{i}-x_{i-1 / 2}} \int_{x_{i-1 / 2}}^{x_{i}} V_{i-1 / 2}\left(\Delta t^{n}, x\right) d x+\frac{1}{x_{i+1 / 2}-x_{i}} \int_{x_{i}}^{x_{i+1 / 2}} V_{i+1 / 2}\left(\Delta t^{n}, x\right) d x .
$$

Notice that we only compute the physical values $\left(H_{i}^{n+1}, u_{i}^{n+1}, Z_{i}^{n+1}\right)$ and that for the conservative quantities $H$ and $Z$ we could also consider a more classical formula by using fluxes at interfaces

$$
H_{i}^{n+1}=H_{i}^{n}-\frac{\Delta t^{n}}{\left|C_{i}\right|}\left(F^{H}\left(V_{i+1 / 2}\left(\Delta t^{n}, x_{i+1 / 2}\right)\right)-F^{H}\left(V_{i-1 / 2}\left(\Delta t^{n}, x_{i-1 / 2}\right)\right)\right) .
$$

\subsection{Solution of the Riemann problem}

The solution of the Riemann problem (10)-(11) will depend on the relative choice of parameters $a$ and $b$. To fix the idea, we suppose in the following that the parameters $a$ and $b$ are such that $a<b$. This requirement is not necessary and some precise bounds on parameters $a$ and $b$ will be given in the last part of this Section. Once this point is clarified, the computation of the eigenvalues of matrix $A$ is now easy and we obtain the following ordered values

$$
u-\frac{b}{H}<u-\frac{a}{H}<u<u+\frac{a}{H}<u+\frac{b}{H}
$$


The associated right eigenvectors are (with $\delta= \pm 1$ )

$$
r_{u+\delta \frac{b}{H}}=\left(\begin{array}{c}
1 \\
\delta \frac{b}{H^{2}} \\
\frac{a^{2}}{H^{2}} \\
\frac{b^{2}-a^{2}}{g H^{3}} \\
\frac{b^{2}-a^{2}}{g H^{3}}\left(u+\delta \frac{b}{H}\right)
\end{array}\right), \quad r_{u+\delta \frac{a}{H}}=\left(\begin{array}{c}
1 \\
\delta \frac{a}{H^{2}} \\
\frac{a^{2}}{H^{2}} \\
0 \\
0
\end{array}\right), \quad r_{u}=\left(\begin{array}{c}
1 \\
0 \\
0 \\
0 \\
0
\end{array}\right) .
$$

We can now compute the solution to the Riemann problem where the relaxation system (5)-(9) is associated to the initial conditions

$$
V(0, x)=\left\{\begin{array}{lll}
V_{l} & \text { if } \quad x<0 \\
V_{r} & \text { if } \quad x \geq 0
\end{array}\right.
$$

where we suppose that the water heights $H_{l}(x)$ and $H_{r}(x)$ are bounded below by a positive constant. The solution contains five waves, each associated to one eigenvalue (13) of the matrix $A$. These five waves separate six constant states that we denote from left to right : $V_{l}, \bar{V}_{l}, V_{l}^{*}, V_{r}^{*}, \bar{V}_{r}$ and $V_{r}$. It follows immediately from the particular form of the right eigenvectors (14) that $Z$ and $\Omega$ are continuous through the three internal waves. Then $\bar{Z}_{l}=Z_{l}^{*}=Z_{r}^{*}=\bar{Z}_{r}$ and we will denote this common value by $Z^{*}$ (resp. $\Omega^{*}$ ). For $u$ and $\Pi$, they are continuous through the intermediate $u$-wave and then $u_{l}^{*}=u_{r}^{*}$ will be denoted by $u^{*}$ (resp. $\Pi^{*}$ ). The twelve remaining unknowns $\left(\bar{h}_{l}, h_{l}^{*}, h_{r}^{*}, \bar{h}_{r}, \bar{u}_{l}, u^{*}, \bar{u}_{r}, \bar{\Pi}_{l}, \Pi^{*}, \bar{\Pi}_{r}, Z^{*}, \Omega^{*}\right)$ are computed by using the continuity of the remaining Riemann invariants through the $a$ - and $b$-waves. For example, through the left $a$-wave (that is associated to the eigenvalue $u-a / h)$, in addition to the continuity of $Z$ and $\Omega$, we have

$$
\bar{u}_{l}-\frac{a}{\bar{h}_{l}}=u^{*}-\frac{a}{h_{l}^{*}}, \quad \bar{\Pi}_{l}+\frac{a^{2}}{\bar{h}_{l}}=\Pi^{*}+\frac{a^{2}}{h_{l}^{*}}
$$

After some computations, it leads to the following relations for the intermediate bottom topography and sediment flux values

$$
Z^{*}=\frac{\left(u_{r}+\frac{b}{H_{r}}\right) Z_{r}-\left(u_{l}-\frac{b}{H_{l}}\right) Z_{l}}{\left(u_{r}+\frac{b}{H_{r}}\right)-\left(u_{l}-\frac{b}{H_{l}}\right)}-\frac{1}{\left(u_{r}+\frac{b}{H_{r}}\right)-\left(u_{l}-\frac{b}{H_{l}}\right)}\left(\Omega_{r}-\Omega_{l}\right),
$$

and

$$
\Omega^{*}=\frac{\left(u_{r}+\frac{b}{H_{r}}\right) \Omega_{r}-\left(u_{l}-\frac{b}{H_{l}}\right) \Omega_{l}}{\left(u_{r}+\frac{b}{H_{r}}\right)-\left(u_{l}-\frac{b}{H_{l}}\right)}-\frac{\left(u_{r}+\frac{b}{H_{r}}\right)\left(u_{l}-\frac{b}{H_{l}}\right)}{\left(u_{r}+\frac{b}{H_{r}}\right)-\left(u_{l}-\frac{b}{H_{l}}\right)}\left(Z_{r}-Z_{l}\right) .
$$

Then we can compute the intermediate water heights

$$
\frac{1}{\bar{H}_{l}}=\left(\frac{1}{H_{l}^{2}}+\frac{2 g}{b^{2}-a^{2}}\left(Z_{l}-Z^{*}\right)\right)^{\frac{1}{2}}, \quad \frac{1}{\bar{H}_{r}}=\left(\frac{1}{H_{r}^{2}}+\frac{2 g}{b^{2}-a^{2}}\left(Z_{r}-Z^{*}\right)\right)^{\frac{1}{2}}
$$


and the intermediate velocities and pressure

$$
\bar{u}_{l}=u_{l}+b\left(\frac{1}{\bar{H}_{l}}-\frac{1}{H_{l}}\right), \quad \bar{u}_{r}=u_{r}-b\left(\frac{1}{\bar{H}_{r}}-\frac{1}{H_{r}}\right),
$$

and

$$
\bar{\Pi}_{l}=\Pi_{l}-a^{2}\left(\frac{1}{\bar{H}_{l}}-\frac{1}{H_{l}}\right), \quad \bar{\Pi}_{r}=\Pi_{r}-a^{2}\left(\frac{1}{\bar{H}_{r}}-\frac{1}{H_{r}}\right) .
$$

We finally compute the internal quantities

$$
\Pi^{*}=\frac{\bar{\Pi}_{l}+\bar{\Pi}_{r}}{2}-\frac{a}{2}\left(\bar{u}_{r}-\bar{u}_{l}\right), \quad u^{*}=\frac{\bar{u}_{l}+\bar{u}_{r}}{2}-\frac{1}{2 a}\left(\bar{\Pi}_{r}-\bar{\Pi}_{l}\right),
$$

and

$$
\frac{1}{H_{l}^{*}}=\frac{1}{\bar{H}_{l}}-\frac{1}{a^{2}}\left(\Pi^{*}-\bar{\Pi}_{l}\right), \quad \frac{1}{H_{r}^{*}}=\frac{1}{\bar{H}_{r}}-\frac{1}{a^{2}}\left(\Pi^{*}-\bar{\Pi}_{r}\right) .
$$

Note that the relaxation model can be extended to the case where one of the water height $H_{l}$ or $H_{r}$ vanishes by adapting the method proposed in [5] for the classical shallow water system.

\subsection{Stability and well-posedness of the relaxation solver}

Before to implement the relaxation solver that we defined in the previous paragraphs, we have to fulfill some basic requirements

- The relaxation model (5)-(9) is stable

- The intermediate states (15)-(21) are well-defined

- The intermediate water heights (17) and (21) are non-negative

Concerning the first point, we first have to define the notion of stability. Here we will consider that the relaxation model is stable if the associated second order system is diffusive. This definition is classical in this context and we refer the reader to [1] for further discussion about this point. To construct the associated second order system, we first introduce an expansion of the auxiliary variables in power of $\lambda$

$$
\Pi=p(H)+\lambda \Pi_{1}+O\left(\lambda^{2}\right), \quad \Omega=Q_{s}(H, u)+\lambda \Omega_{1}+O\left(\lambda^{2}\right)
$$

where $p(H)=g H^{2} / 2$ denotes the fluid pressure. Then we introduce this expansion in the auxiliary equations (7) and (9). This leads to

$$
\begin{aligned}
-\Pi_{1} & =\partial_{t} p(H)+u \partial_{x} p(H)+\frac{a^{2}}{H} \partial_{x} u+O(\lambda) \\
& =p^{\prime}(H) \partial_{t} H+u p^{\prime}(H) \partial_{x} H+\frac{a^{2}}{H} \partial_{x} u+O(\lambda) \\
-\Omega_{1} & =\partial_{t} Q_{s}(H, u)+2 u \partial_{x} Q_{s}(H, u)+\left(\frac{b^{2}}{H^{2}}-u^{2}\right) \partial_{x} z+O(\lambda) \\
& =\partial_{H} Q_{s} \partial_{t} H+\partial_{u} Q_{s} \partial_{t} u+2 u \partial_{H} Q_{s} \partial_{x} H+2 u \partial_{u} Q_{s} \partial_{x} u+\left(\frac{b^{2}}{H^{2}}-u^{2}\right) \partial_{x} z+O(\lambda)
\end{aligned}
$$

Then we use the physical equations (5) and (6) to obtain

$$
\begin{aligned}
-\Pi_{1} & =\frac{1}{H}\left(a^{2}-H^{2} p^{\prime}(H)\right) \partial_{x} u+O(\lambda) \\
-\Omega_{1} & =\left(u \partial_{H} Q_{s}-\frac{p^{\prime}(H)}{H} \partial_{u} Q_{s}\right) \partial_{x} H+\left(-h \partial_{h} Q_{s}+u \partial_{u} Q_{s}\right) \partial_{x} u+\left(\frac{b^{2}}{H^{2}}-u^{2}-g \partial_{u} Q_{s}\right) \partial_{x} Z+O(\lambda)(2)
\end{aligned}
$$


Inserting expansions (22), with particular forms (23)-(24) for the first order correction, in the physical equations (5), (6) and (8) leads to the following second order system

$$
\partial_{t} W+A(W) \partial_{x} W=\lambda \partial_{x}\left(D(W) \partial_{x} W\right)+O\left(\lambda^{2}\right)
$$

with

$$
W=\left(\begin{array}{c}
H \\
u \\
Z
\end{array}\right), \quad D(W)=\left(\begin{array}{ccc}
0 & 0 & 0 \\
0 & a^{2}-H^{2} p^{\prime}(H) & 0 \\
u \partial_{H} Q_{s}-\frac{p^{\prime}(H)}{H} \partial_{u} Q_{s} & -h \partial_{h} Q_{s}+u \partial_{u} Q_{s} & \frac{b^{2}}{H^{2}}-u^{2}-g \partial_{u} Q_{s}
\end{array}\right),
$$

The matrix $D(W)$ is a diffusion matrix if and only if its eigenvalues are nonnegative. It leads to the following lower bounds for parameters $a$ and $b$

$$
a \geq H \sqrt{g H}, \quad b \geq \sqrt{(H u)^{2}+g H^{2} \partial_{u} Q_{s}}
$$

Let us make some comments on these bounds

- The bound on parameter $a$ is the classical bound for relaxation model for barotropic Euler or shallow water equations [21].

- The relative value of $a$ and $b$ depends on the local characteristic values of the flow $(H, u)$ and then it can not be fixed for the whole flow at once, neither in space, nor in time.

- In the low coupling case, the term that involves $Q_{s}$ is small and the relative value of $a$ and $b$ only depends on the nature of the flow : if the flow is fluvial or subsonic $(|u| \leq \sqrt{g H})$, then $a$ has to be larger than $b$ and if the flow is torrential or supersonic, then $b$ has to be larger than $a$.

- In the high coupling case, the parameter $b$ has to be chosen larger than $a$ in the most part of the flow, (possibly) except in the zones where the Froude number is very small.

This first study ensures that the relaxation model is well-posed. In addition we have to be sure that the related relaxation solver is also well-posed and then satisfies the two other requirements : well-posedness of the intermediate states (15)-(21) and non-negativity of the intermediate water heights (17) and (21). Relations (15) and (16) immediately imply

$$
b>\frac{u_{l}-u_{r}}{\frac{1}{h_{l}}+\frac{1}{h_{r}}}
$$

The well-posedness and the positivity of the intermediate water heights leads to more complex relations and it is not so easy to exhibit explicit bounds for $a$ and $b$. Definitions (17) lead to - we recall that we suppose $b>a$

$$
b-a>\max \left(\frac{2 g H_{l}^{2}}{a+b}\left(Z^{*}-Z_{l}\right), \frac{2 g H_{r}^{2}}{a+b}\left(Z^{*}-Z_{r}\right)\right)
$$

Note that this relation is not explicit since the value of $Z^{*}$ depends on the parameter $b$. Moreover definitions (21) need for

$$
a>\max \left(\sqrt{\bar{H}_{l}\left(\Pi^{*}-\bar{\Pi}_{l}\right)}, \sqrt{\bar{H}_{r}\left(\Pi^{*}-\bar{\Pi}_{r}\right)}\right)
$$

Here also the relation is not explicit since the quantities $\bar{H}_{l}, \bar{H}_{r}, \bar{\Pi}_{l}, \bar{\Pi}_{r}$ and $\Pi^{*}$ depends on parameters $a$ and $b$. We do not go further on this point in this note. We only remark that relations (26), (27) and (28) are obviously satisfied for smooth solutions when the space and time steps tend to zero since the right hand sides only involve terms of the form $\Delta u, \Delta Z$ or $\Delta \Pi$. 


\section{Numerical test CASES}

In all the numerical test cases, the parameters $a$ and $b$ of the relaxation solver are chosen such that $a<b$. To ensure this property we impose

$$
a=\max _{i}\left(H_{i} \sqrt{g H_{i}}\right), \quad b=\max \left(\max _{i}\left(\sqrt{\left(H_{i} u_{i}\right)^{2}+g H_{i}^{2} \partial_{u}\left(Q_{s}\right)_{i}}\right), \kappa a\right)
$$

where $\kappa>1$. The other requirements (26)-(28) are not taken into account in the definition of the parameters but are verified a posteriori. Then we apply the solver that is described in the previous section. A less diffusive relaxation solver could be obtained by considering local definitions of parameters $a$ and $b$ and also by locally choosing the adapted relaxation solver, knowing the relative values of $a$ and $b$ that fulfill requirements (25). It needs for the solution of the Riemann problem under the hypothesis $b<a$.

\subsection{Analytical solution}

We first consider a very simple test case for which it is possible to compute an analytical solution [3]. For the Exner law, we consider the Grass formula (1) with $A_{g}=0.005$ and $m=3$. The initial data are the following :

$$
Q(0, x)=Q_{0}, \quad H(0, x)=\frac{Q_{0}}{(\alpha x+\beta)^{1 / 3}}, \quad \frac{Q_{0}^{2}}{2 H(0, x)^{2}}+g(Z(0, x)+H(0, x))=C .
$$

It follows from easy computations, see [3], that this solution is a stationary solution for the fluid part

$$
Q(t, x)=Q(0, x), \quad H(t, x)=H(0, x), \quad \forall t>0,
$$

and that the bottom topography is given by the relation

$$
Z(t, x)=Z(0, x)-A_{g} \alpha t, \quad \forall t>0 .
$$

In Figure 3, we compare the numerical solution with the analytical one for two different number of grid points. We can see that the numerical solution converges to the analytical one. Nevertheless the scheme is quite diffusive since for a small number of point the numerical solution is quite far from the analytical one.

\subsection{Dam break on movable bottom}

We now consider the very classical case of a dam break over a flat bottom but we allow the bottom to evolve in time with respect to the Exner law (4). As initial conditions, the dam is located at $x=5 m$, with $H_{l}=2 \mathrm{~m}$ upstream the dam and $H_{r}=0.125 \mathrm{~m}$ downstream. The topography is initially flat and the flow is at rest $u=0 \mathrm{~m} / \mathrm{s}$. For the Exner law, we still consider the Grass formula (1) with the same parameters and we let evolve in time until $t=1 \mathrm{~s}$, for $J=4000$ cells in space. The results obtained with a splitting approach where the fluid equations and the Exner law are solved separately, presented in Figure 2 in the introduction, exhibit the presence of numerical instabilities (with $J=400$ cells in space). The results obtained with our coupled approach are shown in Figure 4. It shows that for this kind of stiff cases the coupled approach is much more stable. It confirms the conclusion of [7].

\section{3. "Steady" flow over a movable bump}

The last numerical test case is concerned with the extension to movable bottom of the so-called "steady flow over a bump" experiment. Here the flow will not reach a steady state since the bump will be deformed by the 

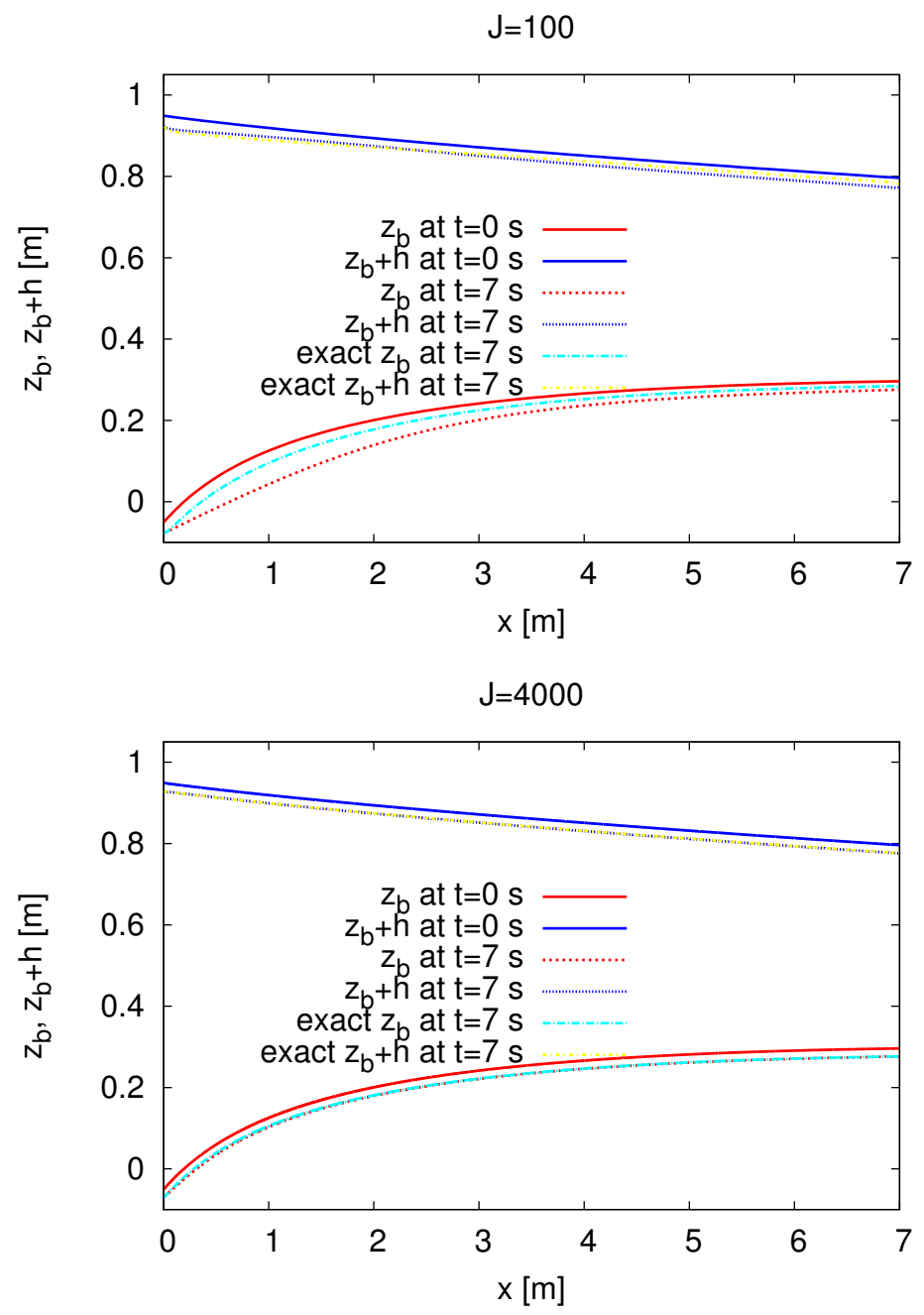

Figure 3. Comparison between numerical and analytical solutions for the Saint-Venant Exner model ; 100 (left) and 4000 (right) grid points

flow. As initial conditions, we consider a subcritical steady state over a bump for the shallow water system as in [7], i.e.

$$
\left\{\begin{array}{l}
Q(x, t=0)=0.5 \\
Z(x, t=0)=0.1+0.1 e^{-(x-5)^{2}} \\
\frac{u^{2}}{2}+g(H+Z)=6.386
\end{array} .\right.
$$

We still consider the Grass formula with the same parameters and we let evolve in time until $t=30 \mathrm{~s}$, for $J=256$ and $J=4000$ cells in space. The results are presented in Figure 5 . With $J=256$ cells, we notice that the scheme is very diffusive. For $J=4000$ cells, we recover the result obtained in [7]. A second order accurate extension of the scheme has to be developed to enhance the precision of the results. We then consider the same initial conditions but with a slightly larger $A_{g}=0.007$, thus the ground is more erodible. The simulation is ended at $t=5 \mathrm{~s}$. It was noticed in [7] that the splitting approach was not adapted for this test case, i.e. they observed some oscillations. Here for $J=4000$ cells, we do not observe any oscillation, see Figure 6 , and the 
Free surface at $\mathrm{t}=1 \mathrm{~s}-\mathrm{J}=4000$

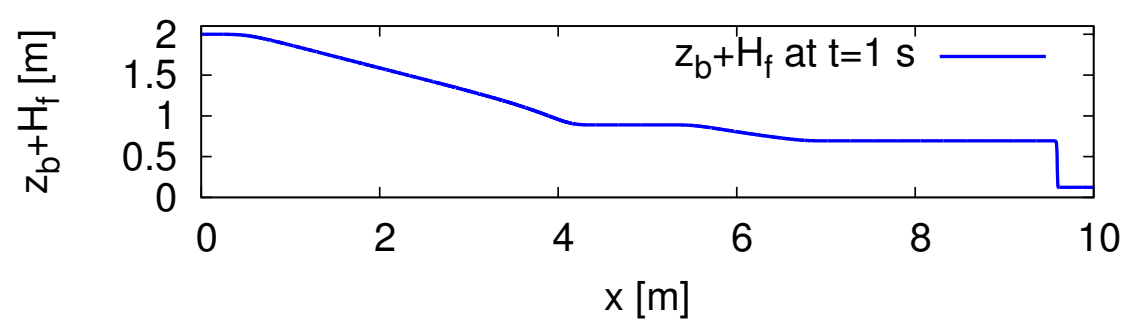

Bottom topography at $\mathrm{t}=1 \mathrm{~s}-\mathrm{J}=4000$

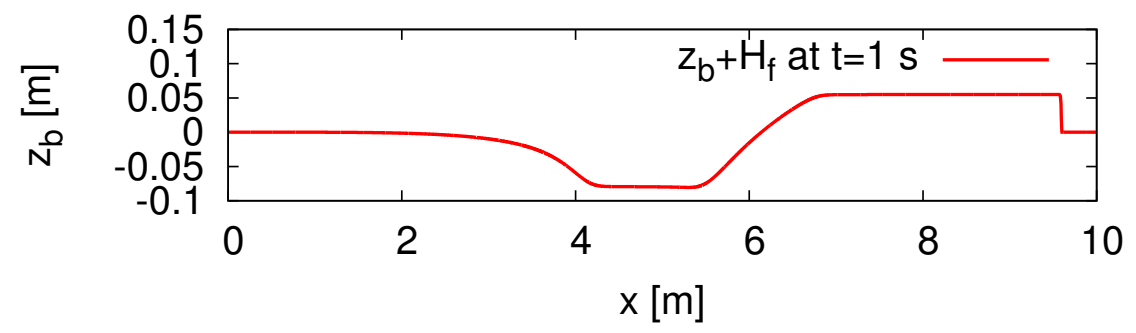

Figure 4. Dam break over a movable bottom - Free surface (top) and bottom topography (bottom)

solution is relevant. This last numerical test confirms the interest of the coupled approach since it is stable for a large class of test cases.
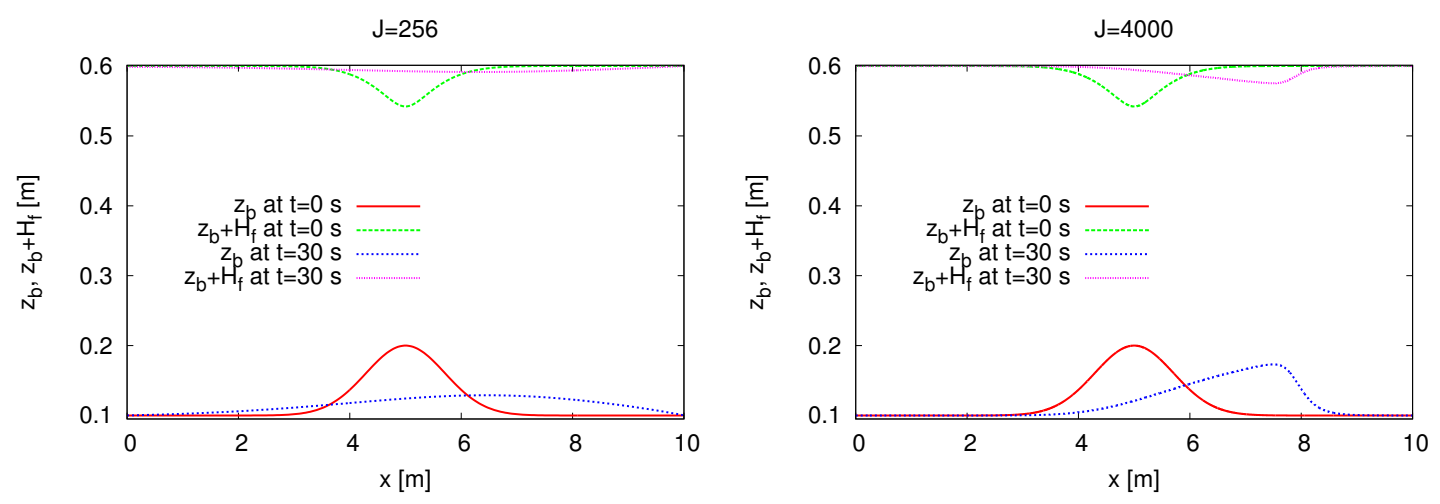

FIGURE 5. Flow over a movable bump, coarse (left) and fine (right) meshes

\section{A two phases Navier Stokes system}

The erosion phenomenon is a fluid/structure interaction problem. It can be formulated in the following way: the displacement of a pure fluid phase that interacts with a movable bed made of a mixture between the pure fluid phase and the static bed components, see Fig 7 . The density of the mixture fluid/solid varies with respect to the concentration of the static bed components. In the following superscripts $b, s$ and $f$ deal respectively with the static bed, mobile bed and pure water layer. We also denote by $H$ the depths of each layer, $u$ their 


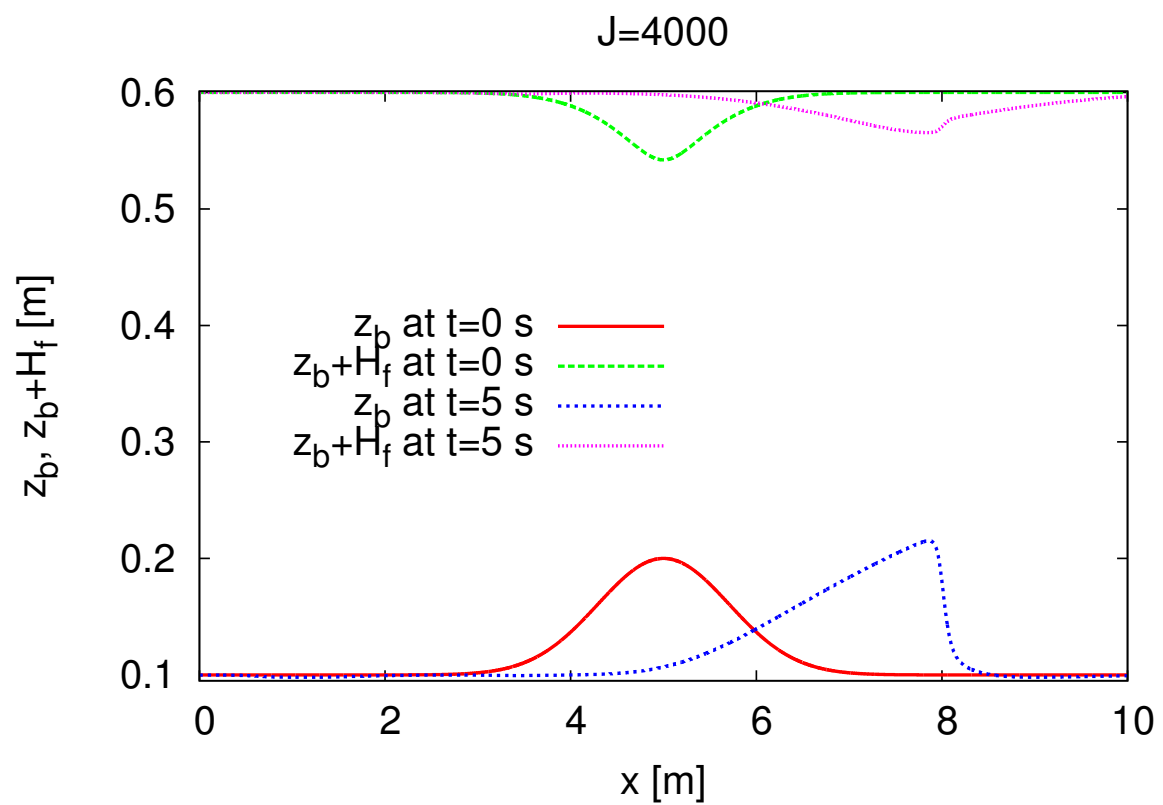

FiguRE 6. Flow over a movable bump, a more erodible bottom $\left(A_{g}=0.007\right)$

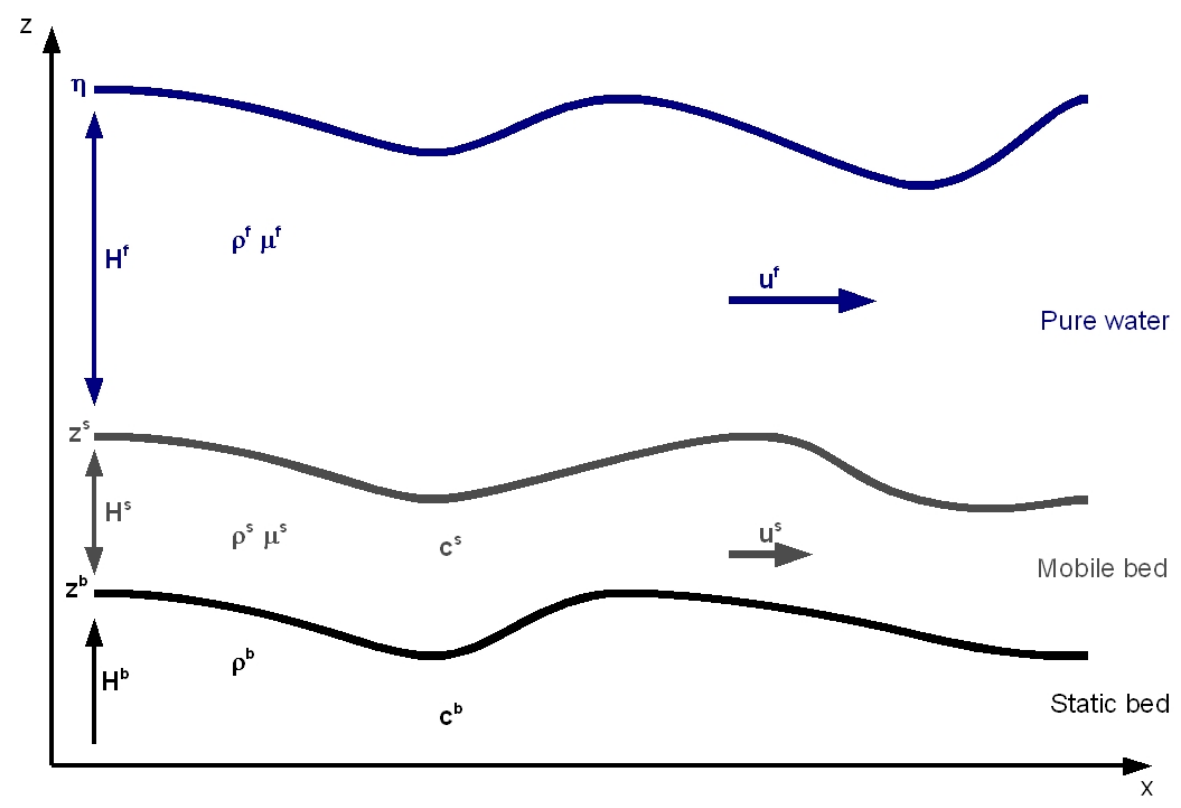

Figure 7. Description of the domain.

mean horizontal velocities, $c$ the sediment concentrations, $\rho$ the mean density, $\mu$ the dynamic viscosity and $g$ the gravity acceleration. The free surface is written $\eta(x, t)$, the bottom topography is $Z(x, t)$ and $z_{s}(x, t)$ stands for the interface between the fluid and the mobile bed. 


\subsection{The fluid part}

We begin by considering the two-dimensional hydrostatic Navier-Stokes system [16] describing a free surface gravitational flow moving over a bottom topography. We denote with $x$ and $z$ the horizontal and vertical directions, respectively. The system has the form

$$
\begin{aligned}
\frac{\partial \rho^{f}}{\partial t}+\frac{\partial \rho^{f} u^{f}}{\partial x}+\frac{\partial \rho^{f} w^{f}}{\partial z} & =0 \\
\frac{\partial \rho^{f} u^{f}}{\partial t}+\frac{\partial \rho^{f}\left(u^{f}\right)^{2}}{\partial x}+\frac{\partial \rho^{f} u^{f} w^{f}}{\partial z}+\frac{\partial p^{f}}{\partial x} & =\frac{\partial \Sigma_{x x}^{f}}{\partial x}+\frac{\partial \Sigma_{x z}^{f}}{\partial z} \\
\frac{\partial p^{f}}{\partial z} & =-\rho^{f} g+\frac{\partial \Sigma_{z x}^{f}}{\partial x}+\frac{\partial \Sigma_{z z}^{f}}{\partial z}
\end{aligned}
$$

and we consider solutions of the equations for, see Fig. 7

$$
t>t_{0}, \quad x \in \mathbb{R}, \quad z_{s}(x, t) \leq z \leq \eta(x, t) .
$$

The chosen form of the viscosity tensor is

$$
\begin{array}{ll}
\Sigma_{x x}^{f}=2 \mu^{f} \frac{\partial u^{f}}{\partial x}, & \Sigma_{x z}^{f}=\mu^{f}\left(\frac{\partial u^{f}}{\partial z}+\frac{\partial w^{f}}{\partial x}\right), \\
\Sigma_{z z}^{f}=2 \mu^{f} \frac{\partial w^{f}}{\partial z}, & \Sigma_{z x}^{f}=\mu^{f}\left(\frac{\partial u^{f}}{\partial z}+\frac{\partial w^{f}}{\partial x}\right) .
\end{array}
$$

The fluid density $\rho^{f}(x, t)$ is assumed to be constant or to depend on the spatial and temporal distribution of the concentration of the sediment $C^{f}(x, t)$ in the fluid layer, namely

$$
\rho^{f}=\rho\left(C^{f}\right)
$$

and $C^{f}$ is governed by a transport-diffusion equation

$$
\frac{\partial \rho^{f} C^{f}}{\partial t}+\frac{\partial \rho^{f} u^{f} C^{f}}{\partial x}+\frac{\partial \rho^{f} w^{f} C^{f}}{\partial z}=\mu_{C}^{f} \frac{\partial^{2} C^{f}}{\partial x^{2}}+\mu_{C}^{f} \frac{\partial^{2} C^{f}}{\partial z^{2}}+S
$$

where $\mu_{C}^{f}$ is the sediment diffusivity and $S$ the source terms (chemical reactions...).

In the previous system we may consider the Boussinesq assumption :

$$
\rho^{f}=\rho_{0}^{f}+\epsilon \rho_{1}^{f}\left(C^{f}\right),
$$

where $\epsilon$ is a small parameter. In that case, at the leading order, the density depends on the concentration only in the gravity term.

\subsection{The mobile bed}

We also consider that the mobile bed can be modelled by the two-dimensional hydrostatic Navier-Stokes system but with a varying density $\rho^{s}$

$$
\begin{aligned}
\frac{\partial \rho^{s}}{\partial t}+\frac{\partial \rho^{s} u^{s}}{\partial x}+\frac{\partial \rho^{s} w^{s}}{\partial z} & =0 \\
\frac{\partial \rho^{s} u^{s}}{\partial t}+\frac{\partial \rho^{s}\left(u^{s}\right)^{2}}{\partial x}+\frac{\partial \rho^{s} u^{s} w^{s}}{\partial z}+\frac{\partial p^{s}}{\partial x} & =\frac{\partial \Sigma_{x x}^{s}}{\partial x}+\frac{\partial \Sigma_{x z}^{s}}{\partial z} \\
\frac{\partial p^{s}}{\partial z} & =-\rho^{s} g+\frac{\partial \Sigma_{z x}^{s}}{\partial x}+\frac{\partial \Sigma_{z z}^{s}}{\partial z}
\end{aligned}
$$


and we consider solutions of the equations for, see Fig. 7

$$
t>t_{0}, \quad x \in \mathbb{R}, \quad Z(x, t) \leq z \leq z_{s}(x, t) .
$$

The viscosity tensor $\Sigma^{s}$ has the same form as in the fluid part. The density $\rho^{s}(x, t)$ is assumed to be constant or to depend on the spatial and temporal distribution of the concentration of the sediment $C^{s}(x, t)$ in the mobile bed, see relations (32) and (33).

\subsection{The boundary conditions}

The systems (29)-(31) and (35)-(37) are completed with boundary conditions. The outward unit normal vector to the free surface $\mathbf{n}_{\eta}$ and the upward unit normal vectors $\mathbf{n}_{f s}$ and $\mathbf{n}_{s b}$ respectively to the interfaces $z_{s}$ and $Z$ are given by

$$
\mathbf{n}_{\eta}=\frac{1}{\sqrt{1+\left(\frac{\partial \eta}{\partial x}\right)^{2}}}\left(\begin{array}{c}
-\frac{\partial \eta}{\partial x} \\
1
\end{array}\right), \quad \mathbf{n}_{j}=\frac{1}{\sqrt{1+\left(\frac{\partial z_{j}}{\partial x}\right)^{2}}}\left(\begin{array}{c}
-\frac{\partial z_{j}}{\partial x} \\
1
\end{array}\right), \quad j=f s, s b .
$$

We then denote $\Sigma_{T}^{j}$ the total stress tensor, which has the form:

$$
\Sigma_{T}^{j}=-p^{j} I_{d}+\left(\begin{array}{cc}
\Sigma_{x x}^{j} & \Sigma_{x z}^{j} \\
\Sigma_{z x}^{j} & \Sigma_{z z}^{j}
\end{array}\right), \quad j=f, s .
$$

\subsubsection{Free surface conditions}

At the free surface we have the kinematic boundary condition

$$
\frac{\partial \eta}{\partial t}+u_{\eta}^{f} \frac{\partial \eta}{\partial x}-w_{\eta}^{f}=0
$$

where the subscript $\eta$ indicates the value of the considered quantity at the free surface.

We denote $\tau_{w}$ the surface wind stress, for which the following expression [14] is considered:

$$
\tau_{w}=C_{D} \rho^{a}\left|V_{w}\right| V_{w},
$$

where $V_{w}$ is the wind velocity, $\rho^{a}$ the air density and $C_{D}$ the wind drag coefficient. Assuming negligible the air viscosity, the continuity of stresses at the free boundary imposes

$$
\Sigma_{T}^{f} \mathbf{n}_{\eta}=-p^{a} \mathbf{n}_{\eta}+\tau_{w} \mathbf{t}_{\eta}
$$

where $p^{a}=p^{a}(x, t)$ is a given function corresponding to the atmospheric pressure and $\mathbf{t}_{\eta}$ is the unit vector orthogonal to $\mathbf{n}_{\eta}$. Relation (39) is equivalent to

$$
\mathbf{n}_{\eta} \cdot \Sigma_{T}^{f} \mathbf{n}_{\eta}=-p^{a}, \quad \mathbf{t}_{\eta} \cdot \Sigma_{T}^{f} \mathbf{n}_{\eta}=\tau_{w} .
$$

In the following, we will assume $p^{a}=0$.

\subsubsection{Boundary conditions at the interface $z_{s}$}

At the interface $z=z_{s}(x, t)$, we impose the continuity of the normal velocity

$$
u_{f s}^{f} \frac{\partial z_{s}}{\partial x}-w_{f s}^{f}=u_{f s}^{s} \frac{\partial z_{s}}{\partial x}-w_{f s}^{s}
$$


and we can define a quantity $e_{f s}$ that describes the exchange between the fluid and the mobile bed, see Fig. 7

$$
e_{f s}=-\frac{\partial z_{s}}{\partial t}-u_{f s}^{f} \frac{\partial z_{s}}{\partial x}+w_{f s}^{f}=-\frac{\partial z_{s}}{\partial t}-u_{f s}^{s} \frac{\partial z_{s}}{\partial x}+w_{f s}^{s}
$$

Moreover the continuity of stresses at the moving boundary $z_{s}$ also imposes the continuity of the pressure and the continuity of tangential stresses

$$
\mathbf{t}_{f s} \cdot \Sigma_{T}^{f} \mathbf{n}_{f s}=\mathbf{t}_{f s} \cdot \Sigma_{T}^{s} \mathbf{n}_{f s}=\tau_{f s}
$$

where $\tau_{f s}$ models inter-phase drag forces which can be expressed as

$$
\tau_{f s}=D\left(H^{f}+H^{s}\right)\left(u_{f s}^{f}-u_{f s}^{s}\right)
$$

where $D=D\left(\left|u_{f s}^{f}-u_{f s}^{s}\right| ; \sigma\right)$ is a drag function depending in general on $\left|u_{f s}^{f}-u_{f s}^{s}\right|$, and a set of physical parameters $\sigma$ (e.g. specific densities, particle diameter), see [19] for a more complete description.

\subsubsection{Boundary conditions at the bottom interface $Z$}

At the interface $z=Z(x, t)$, we have to impose the continuity of the normal velocity (which vanishes since the bottom is supposed to be at rest) and we can define a quantity $e_{f s}$ that describes the exchange between the static and mobile beds, see Fig. 7

$$
e_{s b}=-\frac{\partial Z}{\partial t}
$$

For the stresses at the bottom, we consider a wall law under the form

$$
\mathbf{t}_{s b} \cdot \Sigma_{T}^{s} \mathbf{n}_{s b}=\tau_{b}=\kappa\left(\mathbf{u}_{s b}^{s}, H\right) \mathbf{u}_{s b}^{s} \cdot \mathbf{t}_{s b}
$$

where $\mathbf{t}_{s b}$ is a unit vector satisfying $\mathbf{t}_{s b} \cdot \mathbf{n}_{s b}=0$. Due to thermo-mechanical considerations, in the sequel we will suppose $\kappa\left(\mathbf{u}_{s b}^{s}, H\right) \geq 0$, and $\kappa\left(\mathbf{u}_{s b}^{s}, H\right)$ will be often simply denoted by $\kappa$. If $\kappa\left(\mathbf{u}_{s b}^{s}, H\right)$ is constant then we recover a classical Navier friction condition.

\section{A TWO-PHASE SHALlOW GRANUlaR FLOW MODEL}

\subsection{Derivation}

Let us introduce two small parameters $\varepsilon^{f}$ and $\varepsilon^{s}$ defined by

$$
\varepsilon^{f}=\frac{h^{f}}{\lambda}, \quad \varepsilon^{s}=\frac{h^{s}}{\lambda}
$$

where $\lambda$ is a characteristic dimension along $x$ and $h^{f}, h^{s}$ are characteristic dimensions along the vertical axis in the fluid and mobile bed layers respectively. We consider in this section that the shallow water assumption is valid for the fluid and for the mobile bed. This means the models (29)-(31) and (35)-(37) completed with boundary conditions (38)-(44) can be approximated up to $\mathcal{O}\left(\left(\varepsilon^{f}\right)^{2},\left(\varepsilon^{s}\right)^{2}\right)$ terms by the coupled system (46)-(50) 
Pure Water :

$$
\begin{aligned}
& \frac{\partial H^{f}}{\partial t}+\frac{\partial}{\partial x}\left(H^{f} \bar{u}^{f}\right)=\frac{\rho^{s}}{\rho^{f}} \max \left(0, e_{f s}\right)+\min \left(0, e_{f s}\right) \\
& \frac{\partial\left(H^{f} \bar{u}^{f}\right)}{\partial t}+\frac{\partial}{\partial x}\left(H^{f}\left(\bar{u}^{f}\right)^{2}+\frac{g}{2}\left(H^{f}\right)^{2}\right) \\
& \quad=-g H^{f} \frac{\partial\left(Z+H^{s}\right)}{\partial x}+\frac{\tau_{w}}{\rho^{f}}-\frac{\tau_{f s}}{\rho^{f}}+\frac{\rho^{s}}{\rho^{f}} \bar{u}^{s} \max \left(0, e_{f s}\right)+\bar{u}^{f} \min \left(0, e_{f s}\right),
\end{aligned}
$$

Mobile Bed Layer :

$$
\begin{aligned}
& \frac{\partial H^{s}}{\partial t}+\frac{\partial}{\partial x}\left(H^{s} \bar{u}^{s}\right) \\
& =-\frac{\rho^{f}}{\rho^{s}} \min \left(0, e_{f s}\right)-\max \left(0, e_{f s}\right)+\frac{\rho^{b}}{\rho^{s}} \max \left(0, e_{b}\right)+\min \left(0, e_{b}\right) \\
& \frac{\partial\left(H^{s} u^{s}\right)}{\partial t}+\frac{\partial}{\partial x}\left(H^{s}\left(\bar{u}^{s}\right)^{2}+\frac{\rho^{f}}{\rho^{s}} g H^{s} H^{f}+\frac{g}{2}\left(H^{s}\right)^{2}\right) \\
& =g \frac{\rho^{f}}{\rho^{s}} H^{f} \frac{\partial z_{s}}{\partial x}-g\left(H^{s}+\frac{\rho^{f}}{\rho^{s}} H^{f}\right) \frac{\partial Z}{\partial x}+\frac{\tau_{f s}}{\rho^{s}}-\frac{\tau_{b}}{\rho^{s}} \\
& \quad-\bar{u}_{s} \max \left(0, e_{f s}\right)-\frac{\rho^{f}}{\rho^{s}} \bar{u}_{f} \min \left(0, e_{f s}\right)+\bar{u}_{s} \min \left(0, e_{b}\right),
\end{aligned}
$$

Static Bed Layer :

$$
\frac{\partial Z}{\partial t}=-\max \left(0, e_{s b}\right)-\frac{\rho_{s}}{\rho_{b}} \min \left(0, e_{s b}\right)
$$

This shallow water type model is derived from the original Navier-Stokes equations (29)-(31) by using the shallow water assumption (45) and an integration along the vertical direction. This process is very similar to the one used in [11] to obtain the classical shallow water model. Let us give some details for the fluid layer in the constant density case. First we integrate the mass equation (29) on the fluid layer to obtain

$$
\begin{aligned}
& \frac{\partial}{\partial t} \int_{z_{s}}^{\eta} \rho^{f} d z+\frac{\partial}{\partial x} \int_{z_{s}}^{\eta} \rho^{f} u^{f}(t, x, z) d z \\
& \quad-\rho_{\eta}^{f} \frac{\partial \eta}{\partial t}+\rho_{f s}^{f} \frac{\partial z_{s}}{\partial t}+-\rho_{\eta}^{f} u_{\eta}^{f} \frac{\partial \eta}{\partial x}+\rho_{f s}^{f} u_{f s}^{f} \frac{\partial z_{s}}{\partial x}+\rho_{\eta}^{f} w_{\eta}^{f}-\rho_{f s}^{f} w_{f s}^{f}=0 .
\end{aligned}
$$

Then we use boundary conditions (38) and (41) to write

$$
\frac{\partial}{\partial t} \int_{z_{s}}^{\eta} \rho^{f} d z+\frac{\partial}{\partial x} \int_{z_{s}}^{\eta} \rho^{f} u^{f}(t, x, z) d z+\rho_{f s}^{f} e_{f s}=0 .
$$

Next we introduce the layer depth $H^{f}=\eta-z_{s}$ and the layer velocity

$$
\bar{u}^{f}=\frac{1}{\rho^{f} H^{f}} \int_{z_{s}}^{\eta} \rho^{f} u^{f}(t, x, z) d z,
$$

to obtain

$$
\frac{\partial H^{f}}{\partial t}+\frac{\partial}{\partial x}\left(H^{f} \bar{u}^{f}\right)+\frac{\rho_{f s}}{\rho^{f}} e_{f s}=0
$$


We finally consider an upwind estimation of the interface density $\rho_{f s}$ to derive equation (46). The process is similar for the momentum equation and for the mobile bed layer. Note that comparison between equations (43) and (50) is quite surprising. It should be noted that equation (43) has to be seen as an interface condition between static and mobile bed layers whereas equation (50) is obtained after an integration process over the static bed layer.

In the previous derivation, we assumed that the densities are constant in each layer. The derivation can be done for the general case where the densities depend on the concentration of the sediment in each layer. It is also possible to consider an intermediate version by taking into account the Boussinesq assumption (34).

It remains to give an expression for the exchange terms $e_{f s}$ and $e_{s b}$. In the constant densities case, the total mass conservation imposes a relation between these two parameters

$$
e_{f s}=-\frac{\rho_{b}-\rho_{s}}{\rho_{s}-\rho_{f}} e_{s b}
$$

Possible expressions for $e_{s b}$ are discussed in [20]. Here we choose a relation where $e_{s b}$ is proportional to the jump of the shear stress, and we consider a Mohr-Coulomb bed strength

$$
e_{s b}=\frac{-1}{\rho^{b}\left|u^{s}\right|}\left(\left(\rho^{s}-\rho^{f}\right) H^{s} \tan \phi+\tau_{b}\right), \quad \tau_{b}=C^{b} \rho^{s}\left|u^{s}\right|^{2} \operatorname{sgn}\left(u^{s}\right) . l a b e l e q: \text { esb }
$$

\subsection{Steady states}

For a special choice of constitutive functions for $e_{f s}, e_{s b}, \tau_{f s}, \tau_{b}, \tau_{w}$ and constant densities $\rho^{b}>\rho^{s}>\rho^{f}>0$ of the layers we determine steady states of the problem. Let us first give the missing constitutive relation for the interface shear stress :

$$
\tau_{f s}=C^{s} \frac{\rho^{f}}{\rho^{s}}\left(u^{f}-u^{s}\right)^{2} \operatorname{sgn}\left(u^{f}-u^{s}\right) .
$$

We also consider there is no wind and so $\tau_{w}=0$. To investigate steady states of the system(46)-(50) with the above constitutive laws, we introduce the following expansions, for a small parameter $\varepsilon$ :

$$
H^{i}(x, t)=H_{1}^{i}(x)+\varepsilon H_{1}^{i}(x, t)+o(\varepsilon), \quad u^{i}(x, t)=0+\varepsilon u_{1}^{i}(x, t)+o(\varepsilon), \quad \text { for } i \in\{f, s\}
$$

Then we obtain the following Lemma, whose proof is immediate by inserting the expansions (55), into the equations (46)-(50)

Lemma 5.1. Provided (55) is a solution of (46)-(50) with constitutive laws given by (52)-(54), it satisfies the following conditions

$$
H_{0}^{s}=0, \quad \frac{\partial}{\partial x}\left(H_{0}^{f}+Z\right)=0, \quad H_{1}^{s}=0 .
$$

This shows that we can only have steady states when $H_{0}^{s}$. Thus, the (expected) "lake at rest" condition

$$
\frac{\partial}{\partial x}\left(H_{0}^{f}+H_{0}^{s}+Z\right)=0
$$

is simplified accordingly. The latter condition $H_{1}^{s}=0$ shows that steady states are incompatible even with small (order $\varepsilon$ ) perturbations of $H^{s}$. This indicates some sort of instability of the steady states with respect to this variable. 


\subsection{Linear Stability}

We also tried to determine how the fluxes between the phases in (46)-(50) have to be chosen, such that a linearly stable system is obtained. To this end we started with a simplified model where there is no mass exchange between the phases, the bottom is flat and the pure water and the mobile bed have constant prescribed densities $\rho^{f}, \rho^{s}$, respectively. The only exchange between the phases, we considered, was a strong friction term. As we were not able to determine in which cases the system is linearly stable, we will just explain the problems we encountered, when trying classical linear stability analysis.

The simplified system with quadratic friction reads

$$
\begin{aligned}
\frac{\partial}{\partial t} H^{f}+\frac{\partial}{\partial x}\left(H^{f} u^{f}\right) & =0 \\
\frac{\partial}{\partial t}\left(H^{f} u^{f}\right)+\frac{\partial}{\partial x}\left(H^{f}\left(u^{f}\right)^{2}+\frac{g}{2}\left(H^{f}\right)^{2}\right)+g H^{f} \frac{\partial}{\partial x} H^{s} & =C \lambda^{2}\left|u^{f}-u^{s}\right|\left(u^{f}-u^{s}\right) \\
\frac{\partial}{\partial t} H^{s}+\frac{\partial}{\partial x}\left(H^{s} u^{s}\right) & =0 \\
\frac{\partial}{\partial t}\left(H^{s} u^{s}\right)+\frac{\partial}{\partial x}\left(H^{s}\left(u^{s}\right)^{2}+\frac{g}{2}\left(H^{s}\right)^{2}\right)+r g H^{s} \frac{\partial}{\partial x} H^{f} & =-r C \lambda^{2}\left|u^{f}-u^{s}\right|\left(u^{f}-u^{s}\right)-K \lambda^{2}\left|u^{s}\right| u^{s},
\end{aligned}
$$

where $K \lambda^{2}, C \lambda^{2}$ are friction coefficients and $r:=\rho^{f} / \rho^{s}$. To determine the behavior for strong friction and long times we change the time variable and expanded the velocities

$$
s=\lambda^{-1} t, \quad u^{i}=0+\lambda^{-1} v^{i}+o\left(\lambda^{-1}\right) \quad \text { for } i \in\{f, s\} .
$$

We insert (60) in (56)-(59) and only consider the leading order equations, which are of order $\lambda^{-1}$ for the mass conservation equations and of order $\lambda^{0}$ for the momentum balances. We obtain

$$
\begin{aligned}
\frac{\partial}{\partial s} H^{f}+\frac{\partial}{\partial x}\left(H^{f} v^{f}\right) & =0 \\
\frac{\partial}{\partial x}\left(\frac{g}{2}\left(H^{f}\right)^{2}\right)+g H^{f} \frac{\partial}{\partial x} H^{s} & =C\left|v^{s}-v^{f}\right|\left(v^{s}-v^{f}\right) \\
\frac{\partial}{\partial s} H^{s}+\frac{\partial}{\partial x}\left(H^{s} v^{s}\right) & =0 \\
\frac{\partial}{\partial x}\left(\frac{g}{2}\left(H^{s}\right)^{2}\right)+r g H^{s} \frac{\partial}{\partial x} H^{f} & =-r C\left|v^{s}-v^{f}\right|\left(v^{s}-v^{f}\right)-K\left|v_{s}\right| v_{s} .
\end{aligned}
$$

The equations (61)-(64) can be rewritten as a problem for $H^{s}, H^{f}$ only, by solving (62),(64) for $v^{s}, v^{s}$ and inserting the result into (61),(63). Thereby we obtain

$$
\begin{array}{r}
\frac{\partial}{\partial s} H^{f}+\frac{\partial}{\partial x}\left(H^{f}\left(-\operatorname{sgn}(A) \sqrt{\frac{|A|}{K}}-\operatorname{sgn}\left(H^{f} \frac{\partial}{\partial x}\left(H^{f}+H^{s}\right)\right) \sqrt{\frac{g}{C} H^{f} \frac{\partial}{\partial x}\left(H^{f}+H^{s}\right)}\right)\right)=0 \\
\frac{\partial}{\partial s} H^{s}+\frac{\partial}{\partial x}\left(-H^{s} \operatorname{sgn}(A) \sqrt{\frac{|A|}{K}}\right)=0
\end{array}
$$

where

$$
A:=g r\left(H^{f}+H^{s}\right) \frac{\partial}{\partial x} H^{f}+g\left(r H^{f}+H^{s}\right) \frac{\partial}{\partial x} H^{s} .
$$

To find out whether the system $(65),(66)$ is linear stable or not, one investigates the behavior of perturbations of solution $H_{0}^{s}, H_{0}^{f}$ to $(65),(66)$ as follows. For small values of $\varepsilon$ we define

$$
H^{i}=H_{0}^{i}+\varepsilon H_{1}^{i}+o(\varepsilon) \quad \text { for } i \in\{f, s\}
$$


insert (67) into (65),(66) and study the arising system of equations for $H_{1}^{f}, H_{1}^{s}$. It has the form

$$
\frac{\partial}{\partial s}\left(\begin{array}{c}
H_{1}^{f} \\
H_{1}^{s}
\end{array}\right)+\frac{\partial}{\partial x}\left(\mathcal{A}\left(\begin{array}{c}
H_{1}^{f} \\
H_{1}^{s}
\end{array}\right)+\mathcal{D} \frac{\partial}{\partial x}\left(\begin{array}{c}
H_{1}^{f} \\
H_{1}^{s}
\end{array}\right)\right)=0
$$

where $\mathcal{A}, \mathcal{D}:[0, \infty) \times \mathbb{R} \rightarrow \mathbb{R}^{2 \times 2}$ will be given in the sequel. The classical strategy is to exploit the properties of $\mathcal{A}$ and $\mathcal{D}$ to determine whether or not perturbations are damped over time. This seems to be impossible here because of the involved structure of $\mathcal{A}$ and $\mathcal{D}$. In particular $\mathcal{D}$ is singular for

$$
A_{0}:=g r\left(H_{0}^{f}+H_{0}^{s}\right) \frac{\partial}{\partial x} H_{0}^{f}+g\left(r H_{0}^{f}+H_{0}^{s}\right) \frac{\partial}{\partial x} H_{0}^{s}=0 \quad \text { or } \quad H^{f} \frac{\partial}{\partial x}\left(H_{0}^{f}+H_{0}^{s}\right)=0 .
$$

The latter condition indicates that we cannot consider linearizations around constant solutions, in this setting. Finally let us give the component-wise definitions of $\mathcal{A}$ and $\mathcal{D}$ such that (68) is valid,

$$
\begin{aligned}
& \mathcal{A}_{11}=-\operatorname{sgn}\left(A_{0}\right) \sqrt{\frac{\left|A_{0}\right|}{K}}-\operatorname{sgn}\left(H_{0}^{f} \frac{\partial}{\partial x}\left(H_{0}^{f}+H_{0}^{s}\right)\right) \sqrt{\frac{g}{C} H_{0}^{f} \frac{\partial}{\partial x}\left(H_{0}^{f}+H_{0}^{s}\right)}-\operatorname{sgn}\left(A_{0}\right) H_{0}^{f} \frac{g r}{\sqrt{4\left|A_{0}\right| K}} \frac{\partial}{\partial x}\left(H_{0}^{f}+H_{0}^{s}\right) \\
& -\operatorname{sgn}\left(H_{0}^{f} \frac{\partial}{\partial x}\left(H_{0}^{f}+H_{0}^{s}\right)\right) \sqrt{\frac{g}{4 C H_{0}^{f} \frac{\partial}{\partial x}\left(H_{0}^{f}+H_{0}^{s}\right)}} \frac{\partial}{\partial x}\left(H_{0}^{f}+H_{0}^{s}\right) \\
& \mathcal{A}_{12}=-\operatorname{sgn}\left(A_{0}\right) \frac{g}{\sqrt{4\left|A_{0}\right| K}} H_{0}^{f} \frac{\partial}{\partial x}\left(r H_{0}^{f}+H_{0}^{s}\right), \quad \mathcal{A}_{21}=-\operatorname{sgn}\left(A_{0}\right) \frac{g r}{\sqrt{4\left|A_{0}\right| K}} H_{0}^{s} \frac{\partial}{\partial x}\left(H_{0}^{f}+H_{0}^{s}\right) \\
& \mathcal{A}_{22}=-\operatorname{sgn}\left(A_{0}\right) \sqrt{\frac{\left|A_{0}\right|}{K}}-\operatorname{sgn}\left(A_{0}\right) \frac{g}{\sqrt{4\left|A_{0}\right| K}} H_{0}^{s} \frac{\partial}{\partial x}\left(r H_{0}^{f}+H_{0}^{s}\right) \\
& \mathcal{D}_{11}=-\operatorname{sgn}\left(A_{0}\right) \frac{g r}{\sqrt{4\left|A_{0}\right| K}} H_{0}^{f}\left(H_{0}^{f}+H_{0}^{s}\right)-\operatorname{sgn}\left(H_{0}^{f} \frac{\partial}{\partial x}\left(H_{0}^{f}+H_{0}^{s}\right)\right) \sqrt{\frac{g}{4 C H_{0}^{f} \frac{\partial}{\partial x}\left(H_{0}^{f}+H_{0}^{s}\right)}} H_{0}^{f} \\
& \mathcal{D}_{12}=-\operatorname{sgn}\left(A_{0}\right) \frac{g}{\sqrt{4\left|A_{0}\right| K}} H_{0}^{f}\left(r H_{0}^{f}+H_{0}^{s}\right)-\operatorname{sgn}\left(H_{0}^{f} \frac{\partial}{\partial x}\left(H_{0}^{f}+H_{0}^{s}\right)\right) \sqrt{\frac{g}{4 C H_{0}^{f} \frac{\partial}{\partial x}\left(H_{0}^{f}+H_{0}^{s}\right)}} H_{0}^{f} \\
& \mathcal{D}_{21}=-\operatorname{sgn}\left(A_{0}\right) \frac{g r}{\sqrt{4\left|A_{0}\right| K}} H_{0}^{s}\left(H_{0}^{f}+H_{0}^{s}\right), \quad \mathcal{D}_{22}=-\operatorname{sgn}\left(A_{0}\right) \frac{g}{\sqrt{4\left|A_{0}\right| K}} H_{0}^{s}\left(r H_{0}^{f}+H_{0}^{s}\right) .
\end{aligned}
$$

\section{A Relaxation apProach}

The system (46)-(50) is quite complex : the eigenvalues are impossible to compute analytically and the field are fully non linear. It follows that it is not possible to use approximate Riemann solvers. As in the first part of this work, a possible way to cure this problem is to use a relaxation approach. So we introduce three auxiliary quantities, namely the fluid pressure, the "fluid bottom" and the related "fluid bottom" flux. For simplicity we consider the model with constant densities and we give the details of the method for the upper fluid layer. We first consider the "fluid bottom"

$$
z_{s}(t, x)=Z(t, x)+H^{s}(t, x) .
$$

Using equations (48) and (50), we obtain

$$
\begin{aligned}
\frac{\partial z_{s}}{\partial t}+\frac{\partial}{\partial x}\left(H_{s} \bar{u}_{s}\right)=- & \frac{\rho^{f}}{\rho^{s}} \min \left(0, e_{f s}\right)-\max \left(0, e_{f s}\right)+\frac{\rho^{b}}{\rho^{s}} \max \left(0, e_{b}\right)+\min \left(0, e_{b}\right) \\
& -\max \left(0, e_{s b}\right)-\frac{\rho_{s}}{\rho_{b}} \min \left(0, e_{s b}\right) .
\end{aligned}
$$


Then we can write the following relaxation model for the fluid (we do not consider the zero order right hand side and so we omit the algebraic exchange terms)

$$
\begin{aligned}
\partial_{t} H^{f}+\partial_{x} H_{f} \bar{u}_{f} & =0 \\
\partial_{t} H_{f} \bar{u}_{f}+\partial_{x}\left[H_{f} \bar{u}_{f}^{2}+\pi_{f}\right] & =-g H_{f} \partial_{x} z_{f} \\
\partial_{t} \pi_{f}+\bar{u}_{f} \partial_{x} \pi_{f}+\frac{a_{f}^{2}}{H_{f}} \partial_{x} \bar{u}_{f} & =\frac{1}{\lambda_{f}}\left[g \frac{H_{f}^{2}}{2}-\pi_{f}\right] \\
\partial_{t} z_{f}+\partial_{x} Q_{f} & =\frac{1}{\lambda_{f}}\left[z_{s}-z_{f}\right] \\
\partial_{t} Q_{f}+\left(\frac{b^{2}}{H_{f}^{2}}-\bar{u}_{f}^{2}\right) \partial_{x} z_{f}+2 u_{f} \frac{\partial Q_{f}}{\partial x} & =\frac{1}{\lambda_{f}}\left[H_{s} u_{s}-Q_{f}\right],
\end{aligned}
$$

where $\Pi_{f}$ (resp. $z_{f}$ and $Q_{f}$ ) denotes the auxiliary quantity that allows us to relax the fluid pressure (resp. the "fluid bottom" and "fluid bottom" flux). Here the parameters $a_{f}$ and $b_{f}$ have to be chosen sufficiently large to ensure the stability of the model. Starting from equations (48)-(49) and using equations (46) and (50), we obtain the same kind of model for the mobile bed layer where all the quantities are denoted by subscript $s$

$$
\begin{aligned}
\partial_{t} H_{s}+\partial_{x} H_{s} u_{s} & =0 \\
\partial_{t} H_{s} u_{s}+\partial_{x}\left[H_{s} u_{s}^{2}+\pi_{s}\right] & =-g H_{s} \frac{\partial z_{s}}{\partial x} \\
\partial_{t} \pi_{s}+\bar{u}_{s} \partial_{x} \pi_{s}+\frac{a_{s}^{2}}{H_{s}} \partial_{x} u_{s} & =\frac{1}{\lambda_{s}}\left[g \frac{H_{s}^{2}}{2}-g \frac{\rho_{f}}{\rho_{s}} H_{s} H_{f}-\pi_{s}\right] \\
\partial_{t} z_{s}+\partial_{x} Q_{s} & =\frac{1}{\lambda_{s}}\left[Z+\frac{\rho_{f}}{\rho_{s}} H_{f}-z_{s}\right] \\
\partial_{t} Q_{s}+\left(\frac{b^{2}}{H_{s}^{2}}-\bar{u}_{s}^{2}\right) \partial_{x} z_{s}+2 u_{s} \frac{\partial Q_{s}}{\partial x} & =\frac{1}{\lambda_{s}}\left[\frac{\rho_{f}}{\rho_{s}} H_{f} u_{f}-Q_{s}\right] .
\end{aligned}
$$

Many remarks have now to be done. First, the relaxed model (70)-(78) obviously tends (at least formally) to the original model (46)-(50) when the positive parameters $\lambda_{f}$ and $\lambda_{s}$ tend to zero. Second, we note that the two systems (70)-(74) and (75)-(79) are only coupled through the right hand side relaxation terms that appear in equations (73)-(74) and (77)-(79). Since we see in the first part of this work that these relaxation terms are not explicitely computed (they are taken into account at once when we update the auxiliary quantities at the new time step), it follows that we obtained two decoupled systems! Third, we remark that systems (70)-(74) and (75)-(79) are very similar to system (5)-(9) that we obtained in the first part of this work. It follows that the relaxation solver that has been presented previously can be applied without any big change to this much more complex case.

\section{Conclusion}

In this note, we consider the morphodynamic coupling in rivers. We first propose a coupled finite volume numerical scheme for the classical Saint-Venant - Exner model that is based on a relaxation approach. We prove the relaxation model is stable and the relaxation solver is well-posed and we compute some numerical test cases to show the stability of this new solver even for stiff cases. Then we derived a new three layers model that we hope to be more relevant in the physical sense than the classical approach and we show that the relaxation framework can be extended to this new model. Further work has to be done to improve the properties of the relaxation solver and to extend it to the three-layer model. 


\section{ACKNowledGments}

The authors thank B. Spinewine for discussions about the three-layer model. The authors also thank M.O. Bristeau for her comments that greatly improve the manuscript. O. Delestre, J. Sainte-Marie, G. Sadaka and J. Giesselmann thank EDF for financial support during the CEMRACS. E. Audusse and C. Chalons thank CNRS and INSMI for PEPS financial support. The authors finally greatly thank the CIRM for its warm welcome during the CEMRACS.

\section{REFERENCES}

1. M. Baudin, C. Berthon, F. Coquel, R. Masson, and Q.H. Tran, A relaxation method for two-phase flow models with hydrodynamic closure law, Numer. Math. 99 (2005), 411-440.

2. F. Benkhaldoun, S. Sahmim, and M. Seaid, Solution of the sediment transport equations using a finite volume method based on sign matrix, SIAM J. Sci. Comp. 31 (2009), 2866-2889.

3. C. Berthon, S. Cordier, O. Delestre, and M.H. Le, An analytical solution of shallow water system coupled to exner equation, C. R. Acad. Sci. Paris, Ser. I 350 (2012), 183-186.

4. C. Bertier, J.-P. Bouchard, and L. Dumond, One dimensional model for reservoir sedimentation management, Proceedings of Int. Conf. River Flow (2002), 615-624.

5. F. Bouchut, Nonlinear stability of finite volume methods for hyperbolic conservation laws and well-balanced schemes for sources, Birkhäuser, 2004.

6. M.-J. Castro Diaz, E.-D. Fernandez-Nieto, and A.-M. Ferreiro, Sediment transport models in shallow water equations and numerical approach by high order finite volume methods, Computers \& Fluids 37 (2008), no. 3, 299-316.

7. S. Cordier, H. Le Minh, and T. Morales De Luna, Bedload transport in shallow water models: why splitting (may) fail, how hyperbolicity (can) help, Advances in Water Resources 34 (2011), 980-989.

8. H. A. Einstein, The bed load function for sediment transportation in open channel flows, Technical Bulletin no. 1026, Soil Conservation Service, US Department of Agriculture (1950), 1-71.

9. F. Engelund and J. Fredsoe, A sediment transport model for straight alluvial channels, Nordic Hydrol 7 (1976), $294-298$.

10. F. M. Exner, über die wechselwirkung zwischen wasser und geschiebe in flüssen, Sitzungber. Acad. Wissenscaften Wien Math. Naturwiss. Abt. 134 (1925), 165-180.

11. J.-F. Gerbeau and B. Perthame, Derivation of Viscous Saint-Venant System for Laminar Shallow Water; Numerical Validation, Discrete Contin. Dyn. Syst. Ser. B 1 (2001), no. 1, 89-102.

12. N. Goutal and F. Maurel, A finite volume solver for $1 D$ shallow-water equations applied to an actual river., Int. J. Numer. Meth. Fluids 38 (2002), 1-19.

13. A. J. Grass, Sediments transport by waves and currents, University College, London, Dept. of Civil Engineering, 1981.

14. J. Imberger, Physical processes in lakes and oceans, American Geophysical Union, 1998.

15. S. Jin and Z. Xin, The relaxation schemes for systems of conservation laws in arbitrary space dimensions, Commun. Pure Appl. Math. 48 (1995), 235-276.

16. P.-L. Lions, Mathematical Topics in Fluid Mechanics. Vol. 1: Incompressible models., Oxford University Press, 1996.

17. M. Martin, Modélisation des écoulements à surface libre : étude du ruissellement des eaux de pluie, Rapport de stage de Master 2, Université de Nantes, http://dumas.ccsd.cnrs.fr/dumas-00494243/fr/ (2008), 1-64.

18. E. Meyer-Peter and R. Muller, Formulas for bed-load transport, Proceedings of the 2nd Meeting of the International Association for Hydraulic Structures Research (1948), 39-64.

19. M. Pelanti, F. Bouchut, and A. Mangeney, A riemann solver for single-phase and two-phase shallow flow models based on relaxation. relations with roe and vfroe solvers, J. Comput. Phys. 230 (2011), 515-550.

20. B. Spinewine, Two-layer flow behaviour and the effects of granular dilatancy in dam-break induced sheet-flow, Ph.D. thesis, UCL, 2005.

21. I. Suliciu, Energy estimates in rate-type thermo-viscoplasticity, Int. J. Plast. (1998), 227-244. 